\title{
Research on "Creative" Visual Thinking Mode in Visual Communication Design
}

\author{
Bei Wang ${ }^{1}$ and Mo Wang ${ }^{1}$ \\ Jilin Animation Institute, Changchun, Jilin
}

Keyword: Visual communication design; Creative thinking training

\begin{abstract}
The cultivation of creative thinking is the current topic of design major. In this paper, considering the characteristics and demands of visual design specialty, this dissertation considers how to strengthen the cultivation of creative ability from a multidimensional perspective, has very important reference value for practical work.

With the rapid development of science and technology, visual communication design becomes an indispensable theme of the development of our society in this times. It can provide all kinds of public information for human beings, has a very important aesthetic form, and is also required to be accurate and easy to be understood and accepted. Creative thinking training in this process is of great significance
\end{abstract}

\section{Visual Communication Design and Visual Thinking}

Visual Communication Design. Visual communication design is treating the visual angle as a medium to communicate and release a variety of information. There is a definite purpose in this process, and the information to be conveyed is disseminated through a certain visual art form, which was mainly constituted by illustrations, text and logo the main components.

The characteristics of visual communication is through perceiving the overall visual communication, through people's viewing, association, innovation and other forms to analyze, process and finish clear up. Finally, combining various information to achieve the purpose of clarity and definition of communication.

Visual Thinking. Visual thinking is the way people get the information they need through their own visual thinking, combining with a certain picture, sound, text and other interactive forms to form a certain degree of vivid appeal and rich visual color. This will give full play to the designer's design talent, emphasizing the importance of visual thinking.

Visual thinking is characterized by the establishment of a unique thinking in the human brain, at the same time by using some related aids, such as pictures, text and so on to achieve a more complete visual effects overall. Visible visual thinking is a very positive and perfect action, in this action, we give full play to the ideal positive role.

Creative Visual Thinking. The term of creativity mainly comes from a person's own skills, use of knowledge, creativity and the potential talent. Creativity can be either a relatively static concept of activity to a certain extent or a more dynamic social practice, but both emphasize a certain degree of innovation in the process. To some extent, this kind of thinking can also be seen as a process from scratch. In this process, we emphasize the importance of the word "create", mainly referring to innovation and creation here. While the "meaning" indicates a kind of thinking. Therefore, the creativity requires people to go through a certain degree of meaningful thinking, based on the combination of available and utilized resources, to combine the available resources with their own practical ideas so as to propose a highly feasible solution. To a certain extent, creativity can be interpreted as a reorganization of known things. Based on the more feasible opinions and countermeasures of itself, a more novel and relatively unique thing or something new Concept is designed. 


\section{Analysis of the General Elements of Visual Thinking in Visual Communication Design}

Pay Attention to the Visual Ratio. In the training of visual thinking, the requirement for the proportion element of the picture and text is very clear, the proportion of the size has the impact on the overall visual effects. As the saying goes, there are short and long directors have a certain degree, for the various parts to form visual elements, they must have a clear proportion of the size, too small or larger will have an impact on the vision of the three-dimensional effect, so as not to achieve the desired expression. Therefore, the perfect visual ratio, based on the size, so as to perceive the designer's overall level and unique artistic appreciation, which really give play to the strengths of designer Strengths.

Pay Attention to Personal Subjective Visual Perception. The ultimate goal of visual communication design is to present it to viewers who want to communicate. Therefore, in this process, it is particularly important for the viewer's own personal visual attention. Designers should consider the audience's subjective visual perception of the design and carry out in-depth analysis and research. Because each person's viewpoints on the work of art are different, one of the unchanging rules in this regard is the perception of the first sensations, and the first thing is the overall layout, so from one work we can see that the designers focus on which part, designers should clearly focus on visual design, so as to achieve the overall perception, to achieve a more complete overall perception. In the process of designing a work, the designer not only needs to consider the issue of design from the standpoint of a manufacturer, but also needs to consider empirically and consider the problems from different audience groups so as to achieve a more effective overall perception, design works will be more popular.

Emphasis on Harmony and Unity. Harmony and unity, in the design, are complementary, the two are indispensable. On this basis, it can reflect the different values of the works. This requires that the design of each designer has a unique meaning. The color, layout, structure, lines, the size of these must be considered in the design, there should be a clear arrangement and layout, so that the overall achieve a sense of beauty. To some extent, the art are portrayal of the artist's heart, which requires the designer to have a considerable degree of aesthetic ability, taking full account of the coordination and unity of the various components.

The Perfect Combination of Overall and Part. In appreciation of a piece of work, the viewer first pays attention to the overall effect of a piece of work, and secondly, they should analyze the work of the designer in a partial way and in the analysis of the partial appreciation, it can tests each design unique design skills. This requires designers in the design process must give full consideration to the various elements of mutual coordination, the various parts of the interdependence, to some extent, can be independent of each other, but the overall unity and harmony. This requires the layout of the entire page to be arranged reasonably and overall aesthetic sense achieve harmony and unity, that is to say in the design, we need to take full account of the overall and local coordination, so as to achieve a perfect and coordinated layout.

\section{Analysis of Visual Thinking Creative Elements in Visual Communication Design}

Grasp the Proportion and Size. For the design requirements, one point less or one point more will not reach the perfect level to be expressed. In this process, the focus of attention is still on the scale. Not only does this saying mean that the difference between beauty and non-aesthetic beauty, at the same time, it also emphasizes that the size requirement in design is an important measure of beauty or not.

At the same time, to a certain extent, the size also reflects the relationship between the whole and part, a manifestation of the harmonious design. The perfect ratio and size is an important measure of innovation or not, in the process, it is a standard of reflecting the design level and their own level of self-cultivation.

Emphasis on Personal Visual Center. In a number of studies, it has been found that visual thinking has a clear commonness when it comes to synthesizing feedback on what is viewed internally: interest is central. When viewing a visual work, most of the viewers merely relate to 
works that are of interest to their own vision, illustrating a habit of visual thinking existing in the mind. Therefore, to cultivate creative visual thinking, it is necessary to optimize the visual center so as to achieve a more harmonious relationship between the whole and the part, and partial and constant self-improvement is conducive to the better development of the whole. On the contrary, the continuous improvement of the whole also benefits the better development of the visual center. In the design process, we must continuously carry the training of transposition of the thinking mode, so that the continuous cycle of gradual and orderly, in order to achieve double benefit, not only design works to meet the designer's own design mind, but also they be designed to meet the audience s work.

Coordination and Unity. Only after comparison can we find the obvious difference. In different works of design, the embodiment of artistic value is different. Therefore, the meaning of its presentation and expression is also different. This is the basic means of so-called visual expression design. In the training of innovative thinking, we need to take full account of these differences: the color of the light and dark, the color of the cold and warm, hue differences, shape size, location differences, the number, arrangements tight or close, differences, the location of the distance, height, etc., are the factors designer to consider. Only these factors get a unified arrangement in order to achieve the designer' intention to express the overall beauty. To some extent it can achieves a certain degree of aesthetic significance, thus forming a perception of the overall beauty.

\section{The Cultivation of Creative Thinking in Visual Communication Design}

The Abstract Thinking Training for Naturally Formal Elements. In essence, the training of abstract thinking in the form of essential elements can be regarded as training in the overall perception of vision. This part includes two aspects: one is the reproduction of the essence of the form that the object is to express and the abstract treatment of the two aspects. The training of the essence of the image can make students use a sketch way to ask the students to restore the object's shape, spatial relationship and so on in a tangible, visible and description form, so as to cultivate the students' perception of the formality as a whole. The so-called visual perception; the abstraction of the nature of the image is reflected in a different aspect, which requires that certain objects can be analyzed through training, weakening the relationships between objects, such as space, practice, volume, etc. The relationship between the different constituent elements emphasizes a more abstract thinking ability of essential elements in the expression process.

The Visual Training of Formal Representation Elements. Visual cultivation of formal representational elements is a process of the overall experience of vision. In this process, we must achieve the purpose of expression through the training of the three main elements of the overall three aspects including light and shade, color and texture. In this process, the whole light and shadow can be tested through observation and description, followed by texture training to observe, experience and recognize more effectively, so that to some extent, the sense of touch can be transformed into the sense of visual need, to achieve the necessary sense of perception. Finally, color training, through observation and some tests to develop students' sensitivity to color mastery, to achieve a keen grasp of color.

Comprehensive Training of Creative Visual Thinking. The comprehensive training of creative visual thinking focuses on considering the essential elements of the form itself and conducting continuous comprehensive training on it. So that they have a relatively creative visual perception and a comprehensive experience of the process, so then the two sides are combined with a more rigorous visual induction training. For example, by selecting related pictures through the design requirements, selecting three different images on these pictures and then reassembling the different parts of the cut-off pictures to form a new picture. All these trainings will be useful to well-trained creative visual thinking. Visual communication design emphasizes unrestrained style of presentation, and creative thinking requires divergent thinking as the main way to express it. Therefore, in the whole process of visual communication design, it is particularly important to train creative thinking. It can be seen that to a certain extent, the cultivation of creative thinking is a necessary way for the world to be studied and explored by an unknown art. In this regard, we 
should consider comprehensively from different angles and different aspects. The process reflects the importance of proliferation. Making diffusible innovative thinking become an important part of the world's communication design process. The training content of diffusible thinking mainly includes the diffusion of functions, structures, laws, materials, features, causalities, combinations, words and so on. Therefore, in the process of research, we should consider a variety of different knowledge related to creative thinking, the connection of each knowledge and the value it generates are also regarded as the standard of consideration, which is conducive to the cultivation of the entire visual thinking the study.

\section{References}

[1] Li Wenfang. Visual communication design professional "creative thinking" training mode. Art Education Research, 2015 (4).

[2] Chen Yanjie. The ultimate of design orientation - creative thinking in visual communication design. Journal of Shandong Electric Power College, 2014 (4).

[3] Wang Yanyu. Visual communication design [M]. Beijing: China Light Industry Press, 2011.

[4] Chen Hanmin. Prospect of Art Design Education (I) [M]. Chongqing: Southwest Normal University Press, 2014.

[5] Li Jia. Research on teaching of creative talent cultivation model of art design class in colleges[J]. Packaging World, 2011 (3).

[6] Han Hao. Analysis of the application of Chinese painting ink elements in the design of visual communication in China [J]. Material arts, 2015-08.

[7] Xi Chunbin. Back to nature - visual communication design [J]. Science and Technology Information of Heilongjiang Province; 2014-21 21.

[8] Tao Ye. Visual communication in the space graphics research and application [J]. Journal of Taizhou Vocational and Technical College; 201504.

[9] Zhao Linna. The relationship between Internet and visual communication [J]. Journal of Tangshan Teachers College; 2016-04.

[10]Li Fenghe, Chen Dewei, Xu Bing, Yu Jiawei. Innovative application of folk art in modern visual communication design [J]; Art Sea; 2016-07.

[11] Du Wanjun. Discussion on sense of order in the visual communication design [J]. Popular Literature; 20111616. 